\title{
Lactate Dehydrogenase, Beta-2 Microglobulin Levels and CD4/CD8 Lymphocyte Ratio in Patients with Mycosis Fungoides in Stage 1A
}

\author{
Duygu Gülseren', [MD] \\ ORCID: 0000-0003-1602-726X \\ Ecem Bostan', [MD] \\ ORCID: 0000-0002-8296-4836 \\ Sibel Doğan 1 , [MD] \\ ORCID: 0000-0002-5383-6886
}

Başak YalıcI-Armağan', [MD]

ORCID: 0000-0001-9745-1331

Neslihan Akdoğan', [MD]

ORCID: 0000-0002-1137-5399

Sibel Ersoy-Evans' 1 , [MD]

ORCID: 0000-0002-5040-2476

Gonca Elçin' ${ }^{1},[\mathrm{MD}]$

ORCID: 0000-0001-6292-7272

Ayşen Karaduman', [MD]

ORCID: 0000-0002-4056-6303

Nilgün Atakan', [MD]

ORCID: 0000-0002-8790-1607

'Hacettepe University, Faculty of Medicine, Department of Dermatology and Venereology, Ankara, Turkey.

This article was presented as oral presentation at the 28th National Dermatology Congress held between 24-28 September 2019.

\section{n ABSTRACT Con}

Objective: Mycosis fungoides is the most common type of primary cutaneous T-cell lymphomas. In this study, we aimed to investigate the biochemical parameters of beta-2 microglobulin, lactate dehydrogenase, CD4/CD8 lymphocyte ratio determined by flow cytometry, and T cell clonality in patients with MF and to correlate these parameters in stage $1 \mathrm{~A}$ and other stages.

Materials and Methods: A hundred sixty-one (87 Male, 74 Female) patients followed-up between January 1995 and January 2019 were retrospectively evaluated. Patients' demographics, stage of the disease, peripheral lymph node and organ involvement, peripheral blood beta2-microglobulin, lactate dehydrogenase levels, CD4/CD8 + lymphocyte ratio and presence of $\mathrm{T}$ cell clonality in biopsy samples were evaluated.

Results: Median beta-2 microglobulin levels were $1608.5 \mathrm{ng} / \mathrm{ml}$ for stage $1 \mathrm{~A}$ and $1970.5 \mathrm{ng} / \mathrm{ml}$ for other stages. Peripheral blood median CD4/ CD8 lymphocte ratio was 1.60 for stage $1 \mathrm{~A}$ and 1.85 for other stages. We found statistically significant difference between two groups in terms of beta-2 microglobulin levels and peripheral blood median CD4/CD8 lymphocte ratio $(p=0.001, p=0.04)$. No statistically significant difference was found between two groups in terms of lactate dehydrogenase levels and T-cell clonality $(p=0.234, p=0.525)$.

Conclusion: Our study supports that high peripheral blood beta-2 microglobulin level and CD4/CD8 lymphocyte ratio at the time of diagnosis may imply advanced stage and poor prognosis in Mycosis Fungoides.

Keywords: Cutaneous T-cell lymphoma, Beta-2 microglobulin, CD4/CD8 lymphocyte ratio

Corresponding Author: Duygu Gülseren

Hacettepe University, School of Medicine, Department of Dermatology, Ankara, Turkey.

Phone: +90312305 1704

Fax: +903123097265

E-mail: duygu_gulsrn@hotmail.com

Received: 2 September 2020, Accepted: 24 September 2020,

Published online: 31 December 2020

\section{INRODUCTION}

Mycosis fungoides (MF) is the most common type of primary cutaneous T-cell lymphoma which composes approximately $50 \%$ of primary cutaneous lymphomas. It is characterized by proliferation and occupation of malignant clonal $T$ cell population in the skin [1]. The prognosis of patients with MF is related with the stage and the survival rates decrease in advanced stages. Plaque 
lesions, involvement of more than $10 \%$ of the body surface, altered lactate dehydrogenase (LDH) and beta- 2 microglobulin levels have been found to be associated with disease progression in patients with MF [2].

$\mathrm{LDH}$ is an enzyme which catalyzes the last step of the conversion of pyruvate to lactate during anaerobic glycolysis. Serum LDH is commonly increased in patients with hematopoietic malignancies and it is considered a strong predictor of survival in patients with aggressive lymphoid cancers [3].

Beta-2 microglobulin is a small polypeptide light chain that forms part of the major histocompatibility complex class I antigens. As white-blood-cell membrane turnover is the primary source of serum beta-2 microglobulin, elevated serum beta2 microglobulin levels have been associated with lymphoid malignancies with significant tumour burden, high rates of cellular turnover and more invasive potential [4].

The pathophysiology of MF is thought to be due in part to dysregulated CD4+ T cells, with only approximately $20 \%$ of cases displaying a CD8+ phenotype. In MF, the pathologic CD4+T cell undergoes clonal expansion, potentially leading to abnormally elevated CD4:CD8 T-cell ratio in the peripheral blood [5]

MF is a mature T-cell lymphoma currently thought to develop primarily in the skin by a clonal expansion of a transformed, resident memory $T$ cell (Skin colonization by circulating neoplastic clones in cutaneous T-cell lymphoma). Clonality has not been recorded in skin stage although the detection of a clonal T-cell population in the blood or lymph nodes has been associated with a poorer clinical outcome [6].

In this study, our aim was to investigate the biochemical parameters of beta-2 microglobulin, $\mathrm{LDH}, \mathrm{CD} 4 / \mathrm{CD} 8$ lymphocyte ratio determined by flow cytometry, and the presence of $T$ cell clonality in skin biopsy samples in stage $1 \mathrm{~A}$ and other stages of MF and to show if these parameters are also necessary for stage $1 \mathrm{~A}$ disease. Also we hypothesized that the elevation in the biochemical parameters detected in our patient follow-up may be informative about the progression of the disease.

\section{MATERIALS and METHODS}

After the study protocol was approved by the Hacettepe University School of Medicine ethics committee (2019/06-35), patients applied for the biopsy-proven MF at Hacettepe University School of Medicine, between January 1995 and January 2019 were retrospectively evaluated. Patients with inadequate data were excluded from the study. Patients' demographic data, disease stage at the diagnosis, baseline peripheral blood beta-2 microglobulin, LDH levels, CD4/CD8 lymphocyte ratio and the presence of $\mathrm{T}$ cell clonality in biopsy samples were retrospectively evaluated by using past medical records and electronic data base. Disease stage was determined according to International Society for Cutaneous Lymphomas/ European Organization of Research and Treatment of Cancer (ISCL/EORT) criteria which was proposed in 2007 and staging in patients with a diagnosis of previous years was updated according to the last staging system [7]. Peripheral blood CD4/CD8 lymphocyte ratio was analyzed by flow cytometry and T-cell clonality was evaluated by T-cell receptor (TCR) gene clonal rearrangement analysis in skin biopsy samples.

Statistical analyses were performed with the Statistical Package for the Social Sciences, software version 23.0. (SPSS Inc., Chicago, IL, USA). Numerical variables were summarized as mean \pm standard deviation or median (minimum-maximum). Numerical variables were compared by MannWhitney $U$ test and categorical variables were given as frequencies and percentages. Categorical variables were compared by Chi-square test. A value of $P<0.05$ was considered statistically significant.

\section{RESULTS}

Totally, 161 patients with the diagnosis of MF were included in the study. Seventy-four (46\%) patients were female and 87 (54\%) were male. The mean age was $45.9 \pm 15.56$ years (range: $12-84$ years). The distribution of the dates of diagnosis was given in Figure 1. The distribution of the disease stages was shown in Figure 2. Stage $1 \mathrm{~A}$ disease was detected mostly and it was in 106 (65.8\%) patients. Due to the lack of homogenous patient distribution according to disease stage, groups were categorized as stage $1 \mathrm{~A}$ and other stages. 


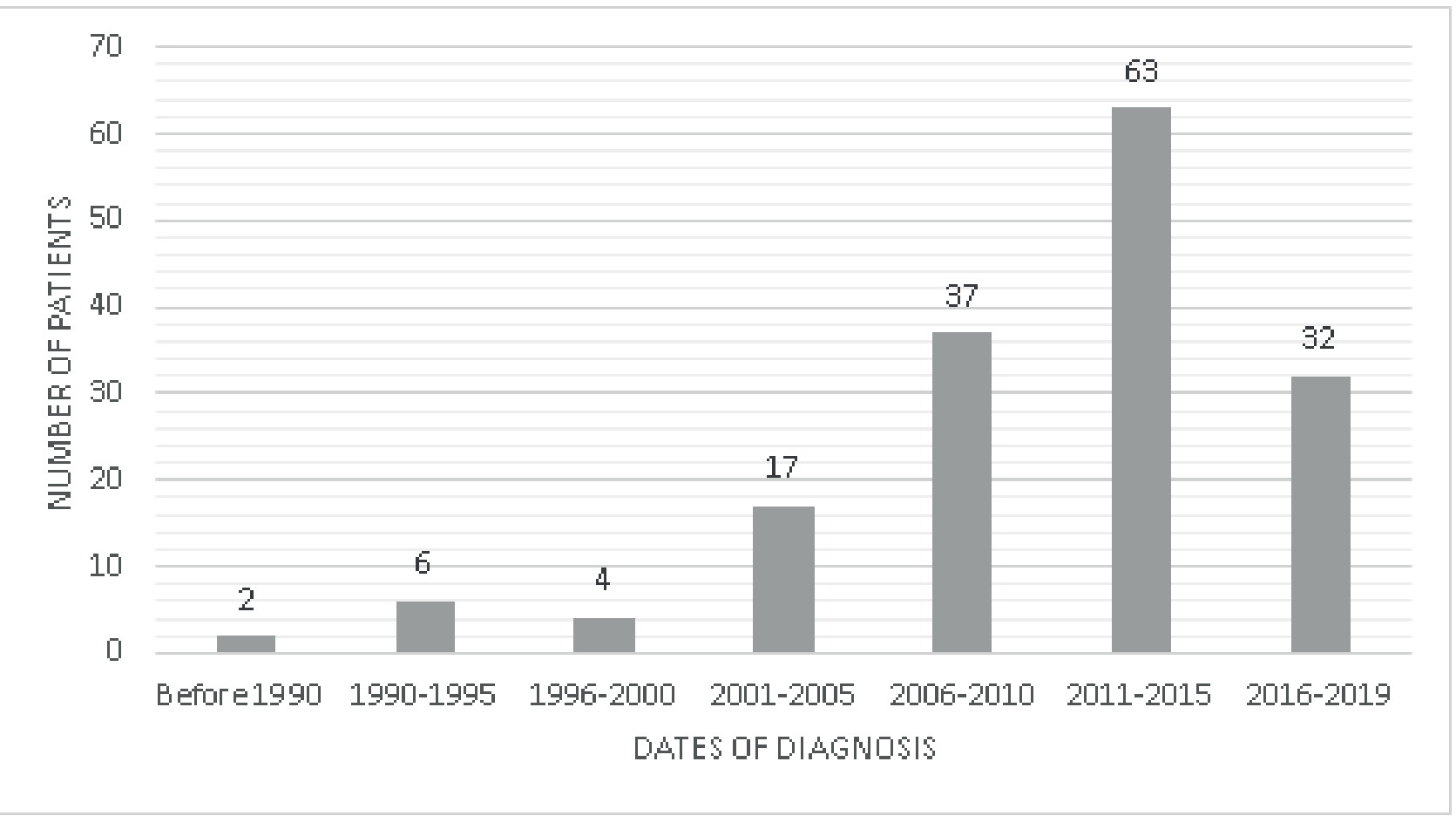

Figure 1. The distribution of the dates of diagnosis

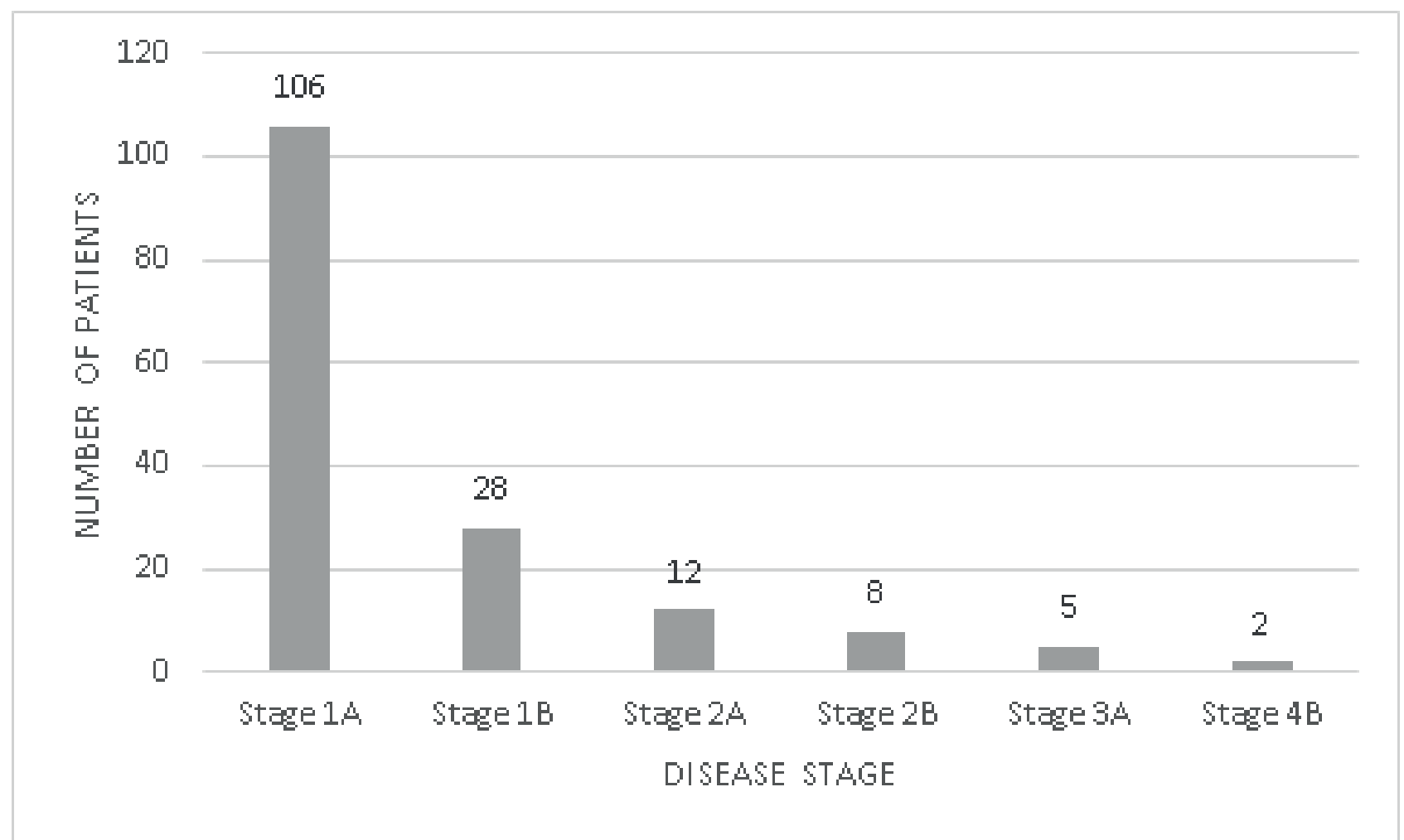

Figure 2. The distribution of the disease stages

Lymph node involvement was found in 19 (11.8\%) patients and internal organ involvement was only in $2(1.2 \%)$ patients. Median LDH and beta-2 microglobulin levels for stage $1 \mathrm{~A}$ were respectively 273.0 U/L (range: $123-548$ ) and $1608.5 \mathrm{ng} / \mathrm{ml}$ (range: 941-4595). Median LDH and beta-2 microglobulin levels for other stages were respectively $318 \mathrm{U} / \mathrm{L}$ (range:130-855) and $1970.5 \mathrm{ng} / \mathrm{ml}$ (range: 94111021). We only found statistically significant difference in terms of beta-2 microglobulin levels between two groups ( $p=0.001$ ). Median CD4/CD8 lymphocyte ratio was 1.60 (range: $0.59-6.60$ ) for stage $1 \mathrm{~A}$ and 1.85 (0.70-17.40) for other stages (Table 1). There was statistically significant difference 
Table 1. Biochemical Parameters according to disease stages

\begin{tabular}{|c|c|c|c|c|}
\hline PARAMETERS & TOTAL & STAGE 1A & STAGE 1B-4 & PVALUE \\
\hline LDH (U/L) & & & & 0.234 \\
\hline Available Count & 155 & 101 & 54 & \\
\hline Mean & 295.66 & 280.67 & 323.70 & \\
\hline Standard Deviation & 126.25 & 100.78 & 160.99 & \\
\hline Median & 278 & 273 & 318 & \\
\hline Range & $123-855$ & $123-548$ & $130-855$ & \\
\hline BETA-2 MICROGLOBULIN (ng/mL) & & & & 0.001 \\
\hline Available Count & 152 & 100 & 52 & \\
\hline Mean & 2017.95 & 1732.21 & 2567.44 & \\
\hline Standard Deviation & 1198.01 & 537.72 & 1794.49 & \\
\hline Median & 1676.50 & 1608.50 & 1970.50 & \\
\hline Range & $941-11021$ & $941-4595$ & 1166-11021 & \\
\hline Peripheral CD4/CD8 lymphocyte ratio & & & & 0.040 \\
\hline Available Count & 138 & 88 & 50 & \\
\hline Mean & 2.01 & 1.76 & 2.43 & \\
\hline Standard Deviation & 1.83 & 1.01 & 2.70 & \\
\hline Median & 1.64 & 1.60 & 1.85 & \\
\hline Range & $0.59-17.40$ & $0.59-6.60$ & $0.70-17.40$ & \\
\hline
\end{tabular}

between two groups in terms of peripheral blood $C D 4 / C D 8$ lymphocyte ratio $(p=0.04)$. TCR gene rearrangement results were available only in 111 (68.9\%) patients; $40(5.6 \%)$ of 76 patients with stage $1 \mathrm{~A}$ and $20(57.1 \%)$ of 35 patients with other stages had proven T-cell clonality. No statistically significant difference was found between two groups in term of T-cell clonality $(p=0.525)$.

\section{DISCUSSION}

In our study, we aimed to show the correlation between biochemical parameters that are frequently used for baseline screening of MF patients and disease stage. We wanted to show if detailed biochemical parameters are necessary for stage $1 \mathrm{~A}$ disease. We only found statistically significant difference in beta- 2 microglobulin and CD4/CD8 lymphocyte ratio between stage $1 \mathrm{~A}$ and other stages.

Beta-2 microglobulin is a low molecular weight $(17 \mathrm{kDa})$ protein found in all nucleated cells and body fluids such as serum, cerebrospinal fluid and urine [8]. Amorim et al [2] showed that altered LDH and beta-2 microglobulin levels were significantly associated with disease progression and higher frequency of deaths in MF patients. Similar to previous findings in the literature, our results support that patients with other stages have higher beta- 2 microglobulin levels than stage $1 \mathrm{~A}$ patients who have better prognosis.

LDH is a routinely used biochemical marker in solid and hematological cancer patients to determine tumor burden, progression, prognosis and response to treatment. In order to proliferate efficiently and rapidly tumor cells mainly go through glycolysis to supply energy [9]. As shown in solid organ tumors and hematological cancers, prognostic significance of $\mathrm{LDH}$ is also demonstrated in MF patients. Serum $\mathrm{LDH}$ level is found to be positively correlated with advanced stage in MF patients and asserted to be used as an independent risk factor for disease progression [10]. In our study, we found higher LDH levels in patients with other stages but it was statistically non-significant. The low number of patients with other stages than $1 \mathrm{~A}$ may be the reason for not being able to show this association.

Peripheral blood CD4/CD8 lymphocyte ratio which shows circulating lymphocyte subsets is found to be prognostically important parameter to predict the efficacy and clinical response to electron beam radiation therapy in patients with MF, higher levels of CD4/CD8 implying poor prognosis and response to therapy [11]. Like previous studies in the literature, our patients with other stages has higher peripheral blood CD4/CD8 lymphocyte ratio than stage $1 \mathrm{~A}$ patients. 
In our study, we analyzed the results of TCR gene clonal rearrangement analysis which are shown to be a useful tool to aid in the early diagnosis of MF [12]. Even though, detection of clonal T cells may be utilized to support the diagnosis of MF, however, the presence of non-clonal T-cells does not exclude the diagnosis of MF in the existence of other supportive, clinical, histopathological and immunochemical criteria [13]. In our study, we were not able to find any significant difference between stage $1 \mathrm{~A}$ and other stages in terms of T-cell clonality in biopsy specimen. This data might suggest that T-cell clonality is a useful parameter for the diagnosis of MF but it cannot be an indicator of stage or prognosis.

The limitations of the study are the lack of homogenous patient distribution according to disease stage. This is a retrospective study and missing data cannot be analyzed. As the study covers a long period of 24 years, blood sampling time, sampling method, sample transport, and sample storage cannot be done under the same conditions.
In conclusion, our study supports that high peripheral blood beta-2 microglobulin level and CD4/CD8 lymphocyte ratio at the time of diagnosis may imply advanced stage and poor prognosis. The higher beta-2 microglobulin level and CD4/CD8 lymphocyte ratio we detected in advanced stage patients are also important data for the followup of the patients and these parameters can be informative about the progression of the disease. T-cell clonality might be needed to diagnose of MF but it is not an indicator of advanced stage.

\section{ACKNOWLEDGEMENTS}

We thank Sevilay Karahan, MD for statistical analysis of the study data.

\section{CONFLICT Of INTEREST STATEMENT}

The authors declare that they have no conflict of interest. 
[1] Girardi M, Heald PW, Wilson LD. The pathogenesis of mycosis fungoides. N Engl J Med. 2004; 350: 1978-88.

[2] Amorim GM, Niemeyer-Corbellini JP, Quintella DC, et al. Clinical and epidemiological profile of patients with early stage mycosis fungoides. An Bras Dermatol. 2018; 93: 54652.

[3] Terragni R, Morselli-Labate A, Massimo Vignoli $M$, et al. Is Serum Total LDH Evaluation Able to Differentiate between Alimentary Lymphoma and Inflammatory Bowel Disease in a Real World Clinical Setting? PLoS One. 2016; 11: e0151641

[4] Bento L, Díaz-López A, Barranco G, et al. New prognosis score including absolute lymphocyte/monocyte ratio, red blood cell distribution width and beta-2 microglobulin in patients with diffuse large B-cell lymphoma treated with R-CHOP: Spanish Lymphoma Group Experience (GELTAMO). Br J Haematol. 2020; 188: 888-97.

[5] An $Y$, Jiang $W$, Andraos TY, et al. Multi-institutional Investigation: Circulating CD4:CD8 ratio is a prognosticator of response to total skin electron beam radiation in mycosis fungoides. Radiother Oncol. 2019; 131: 88-92

[6] Massone C, Crisman G, Kerl H, et al. The prognosis of earlymycosis fungoides is not influenced by phenotype and T-cell clon-ality. Br J Dermatol 2008; 159: 881-6.

[7] Olsen E, Vonderheid E, Pimpinelli N, et al. Revisions to the staging and classification of mycosis fungoides and Sezary syndrome: a proposal of the International Society for Cutaneous Lymphomas (ISCL) and the cutaneous lymphoma task force of the European Organization of Research and Treatment of Cancer (EORTC). Blood 2007; 110: 1713-22.

[8] Güssow D, Rein R, Ginjaar I, et al. The human beta 2-microglobulin gene. Primary structure and definition of the transcriptional unit. J Immunol. 1987; 139: 3132-8.

[9] Chen ZH, Qiu MZ, Wu XY, et al. Elevated baseline serum lactate dehydrogenase indicates a poor prognosis in primary duodenum adenocarcinoma patients. J Cancer. 2018; 9: 512-20.

[10] Saif FA. Prognostic Significance of Serum Lactate Dehydrogenase in Saudi Patients with Mycosis Fungoides: A Retrospective Study of 47 Patients. J Clin Exp Dermatol Res. 2016; 7: 2 .

[11] Jiang, W, Abou Yehia, Z, Dabaja B, et al. CD4:CD8 Ratio in Mycosis Fungoides: Prognostic and Predictive Value of Circulating T Lymphocyte Subsets for Outcomes After Total Skin Electron Beam Radiation. International Journal of Radiation Oncology, Biology, Physics. 2016; 96: S20.

[12] Xu C, Wan C, Wang L, et al. Diagnostic significance of TCR gene clonal rearrangement analysis in early mycosis fungoides. Chin J Cancer. 2011; 30: 264-72.

[13] Acikalin A, Bagir E, Tuncer I, et al. Contribution of T-cell receptor gamma gene rearrangement by polymerase chain reaction and immunohistochemistry to the histological diagnosis of early mycosis fungoides. Saudi Med J. 2013; 34: 19-23. 